EUROPEAN ORGANIZATION FOR NUCLEAR RESEARCH

CERN - AB Department

CERN-AB-2007-046

\title{
DESIGN AND PERFORMANCE OF THE CNGS SECONDARY BEAM LINE
}

E. Gschwendtner, L. Bruno, K. Elsener, A. Ferrari, A. Guglielmi ${ }^{1}$, M. Medahi, A. Pardons, S. Rangod, P. Sala ${ }^{2}$

CERN, Geneva, Switzerland

\begin{abstract}
An intense muon-neutrino beam $\left(10^{17} v_{\mu} /\right.$ day $)$ is generated at CERN and directed towards the Gran Sasso National Laboratory, LNGS, in Italy, $732 \mathrm{~km}$ away from CERN.

In the presently approved physics programme, it is foreseen to run the CNGS facility with $4.510^{19}$ protons per year for five years. During a nominal CNGS cycle, i.e. every 6s, two nominal SPS extractions of $2.410^{13}$ protons each at $400 \mathrm{GeV} / \mathrm{c}$ are sent down the proton beam line to the target.

The CNGS secondary beam line, starting with the target, has to cope with this situation, which pushes the beam line equipment and instrumentation to the limits of radiation hardness and mechanical stresses during the CNGS operation.

An overview of the CNGS secondary beam line is given. Emphasis is on the target, the magnetic focusing lenses (horn and reflector) and the muon monitors. The performance of the secondary beam line during beam commissioning and physics operation is discussed and measurements are compared with simulations.
\end{abstract}

\footnotetext{
${ }^{1)}$ INFN Padova, Italy

2) INFN Milano, Italy
}

Presented at

PAC07, 22nd PAC Conference, June 25-29, 2007, Albuquerque, USA

Geneva, Switzerland

August 2007 


\title{
DESIGN AND PERFORMANCE OF THE CNGS SECONDARY BEAM LINE
}

\author{
E. Gschwendtner, L. Bruno, K. Elsener, A. Ferrari, M. Meddahi, A. Pardons, S. Rangod, \\ CERN Geneva Switzerland; A. Guglielmi, INFN Padova Italy; P. Sala, INFN Milano Italy
}

\begin{abstract}
An intense muon-neutrino beam $\left(10^{17} v_{\mu} /\right.$ day $)$ is generated at CERN and directed towards the Gran Sasso National Laboratory, LNGS, in Italy, $732 \mathrm{~km}$ from CERN.

In the presently approved physics programme, it is foreseen to run the CNGS facility with $4.5 \cdot 10^{19}$ protons per year for five years. During a nominal CNGS cycle, i.e. every $6 \mathrm{~s}$, two nominal SPS extractions of $2.4 \cdot 10^{13}$ protons each at $400 \mathrm{GeV} / \mathrm{c}$ are sent down the proton beam line to the target.

The CNGS secondary beam line, starting with the target, has to cope with this situation, which pushes the beam line equipment and instrumentation to the limits of radiation hardness and mechanical stresses during the CNGS operation.

An overview of the CNGS secondary beam line is given. Emphasis is on the target, the magnetic focusing lenses (horn and reflector) and the muon monitors. The performance of the secondary beam line during beam commissioning and physics operation is discussed and measurements are compared with simulations.
\end{abstract}

\section{INTRODUCTION}

A schematic overview of the CNGS neutrino beam facility [1] at CERN is shown in Figure 1. $400 \mathrm{GeV} / \mathrm{c}$ protons extracted from the SPS accelerator are sent down the proton beam line onto a carbon target, producing pions and kaons. The positively charged $\pi / \mathrm{K}$ are energyselected and guided with two focusing lenses, the socalled horn and reflector, in the direction towards Gran Sasso. These particles decay in a $1000 \mathrm{~m}$ long decay vacuum tube into muon-neutrinos and muons. All the hadrons, i.e. protons that have not interacted in the target, pions and kaons that have not decayed in flight, are absorbed in a hadron stopper. Only neutrinos and muons can traverse this $18 \mathrm{~m}$ long block of graphite and iron. The muons, which are ultimately absorbed downstream in around $500 \mathrm{~m}$ of rock, are measured in two muon detector stations. This allows concluding on the intensity of the neutrino beam produced and on the beam profile.

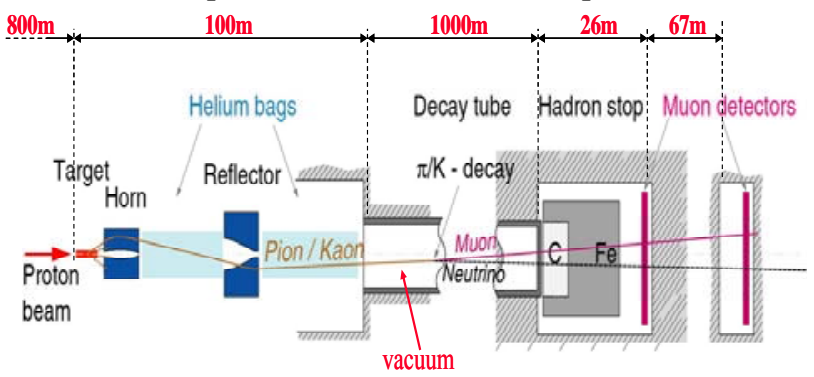

Figure 1: Main components of CNGS.

\section{THE SECONDARY BEAM LINE}

The proton (primary) beam line running from the SPS extraction to the target is described in [2]. The secondary beam line reaches from the CNGS target to the muon monitors.

\section{CNGS Target}

The CNGS target is designed to withstand the beam induced stress (thermo mechanical shocks, heat deposit) up to $3.5 \cdot 10^{13}$ protons per extraction with $400 \mathrm{GeV} / \mathrm{c}$ (up to $750 \mathrm{~kW}$ average power) [3, 4]. Figure 2 shows the CNGS target magazine, consisting of five target units. One unit is used in the beam; the other four are in-situ spares. Each target unit consists of 13 graphite rods which are $10 \mathrm{~cm}$ long and interspaced by $9 \mathrm{~cm}$. The diameter of the first two rods is 5mm; the other rods have a diameter of $4 \mathrm{~mm}$. The rods are supported by a carbon composite tube, which in turn is inserted in an aluminium tube with beryllium windows providing a static sealed system filled with 0.5 bar of helium gas (one spare target unit is left under vacuum). The target magazine as well as the surrounding iron shielding is air-cooled.

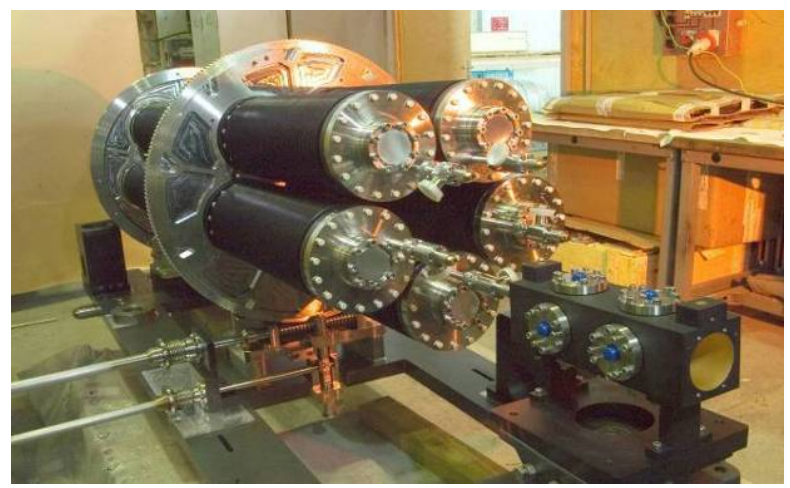

Figure 2: CNGS target magazine consisting of five target units. The bottom unit intercepts the proton beam, the other four units are spares.

\section{CNGS Horns}

The magnetic focusing system, comprising the horn and reflector (each $7 \mathrm{~m} \mathrm{long}$ ), is installed downstream of the target. The horn and reflector are toroidal lenses with a focusing magnetic field between the inner and outer conductor, created by a pulsed high current. The shape of the inner conductor provides the desired focusing for particles with a wide range of momenta and angles. The focused particle momentum range is 20 to $50 \mathrm{GeV} / \mathrm{c}$. The horn (Figure 3 ) is pulsed twice every 6 s cycle with a current of $150 \mathrm{kA}$ during a few milliseconds, the reflector with $180 \mathrm{kA}$. The two pulses are separated by $50 \mathrm{~ms}$, synchronized with the two beam pulses. The water 
cooling systems of the horn is designed for a proton intensity of $7.2 \cdot 10^{13}$ protons per $6 \mathrm{~s}$ cycle $\left(3.6 \cdot 10^{13}\right.$ protons/extraction) at $400 \mathrm{GeV} / \mathrm{c}$ [5]. The corresponding total power to be evacuated in the horn is $26 \mathrm{~kW}$, coming from the particle interactions in the horn and the applied current. The principal characteristics of horn and reflector are summarized in Table 1.

Special features are implemented on the cavern crane and the CNGS equipment in order to exchange the target or the horn remotely with spare ones in case of a failure. A limited number of manual interventions are nevertheless needed before remote exchange operation [6].

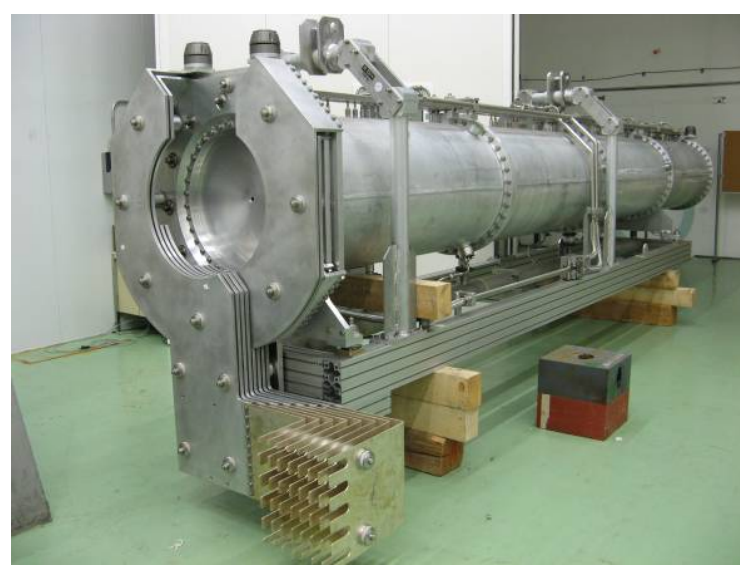

Figure 3: The CNGS horn, before installation in the target cavern.

Table 1: Horn and reflector characteristics.

\begin{tabular}{|l|l|l|l|}
\hline & Unit & $\begin{array}{l}\text { Horn } \\
\text { system }\end{array}$ & $\begin{array}{l}\text { Reflector } \\
\text { system }\end{array}$ \\
\hline Load peak current & $\mathrm{kA}$ & 150 & 180 \\
\hline Pulse duration & $\mathrm{ms}$ & 6.5 & 9.8 \\
\hline Transformer ratio & & 16 & 32 \\
\hline Primary peak current & $\mathrm{A}$ & 9375 & 5646 \\
\hline Capacitor charging voltage & $\mathrm{V}$ & 6300 & 5800 \\
\hline Water flow for $\Delta \mathrm{T}=5^{\circ} \mathrm{C}$ & $1 / \mathrm{min}$ & 50 & 50 \\
\hline Water pressure & Bar & 1.2 & 1.2 \\
\hline
\end{tabular}

\section{Muon Monitors}

The two muon detector stations provide on-line feedback for the quality control of the neutrino beam. The muon detector stations are installed downstream of the hadron stopper and are separated by $67 \mathrm{~m}$ of rock. The detectors are designed to measure up to $10^{8}$ muons $/ \mathrm{cm}^{2}$ per extraction, i.e. over $10.5 \mu$ s. To cope with such a high instantaneous rate nitrogen-filled, sealed ionization chambers are used. Each detector has 64 electrodes separated by $5 \mathrm{~mm}$. The electrode diameter is $4 \mathrm{~cm}$ and the bias voltage is set to 800 Volts [7]. In each of the two CNGS muon detector chambers, there are 41 fixed muon detectors installed. They are assembled in a cross-shaped array (see Figure 4) to provide the muon intensity and the vertical and horizontal muon profile. In addition an identical monitor is installed on a motorized support, downstream of the fixed ones to allow cross-calibration of the fixed monitors and to probe the muon profile where there is no fixed monitor.

The muon energy spectrum cut-off is different in the two chambers. Only muons with energies above $\sim 20 \mathrm{GeV} / \mathrm{c}$ $(\sim 50 \mathrm{GeV} / \mathrm{c})$ reach the first (second) muon detector station.
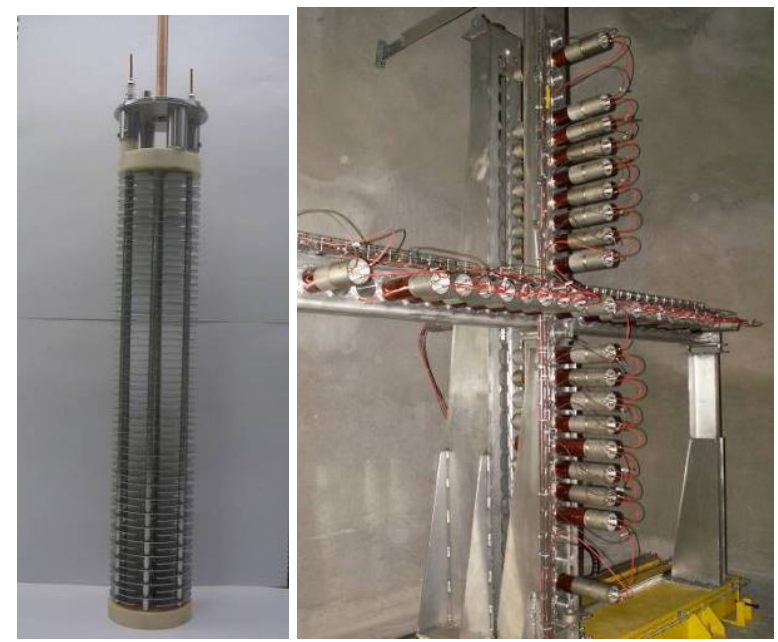

Figure 4: Left: a single muon detector, without its stainless steel casing. Right: A muon detector station with the muon detectors arranged in a cross-shaped array.

\section{SECONDARY BEAM LINE PERFORMANCE}

The performance of the CNGS beam has been extensively checked during the commissioning of the CNGS facility in a three-week period in July/August 2006 and during the 10 day CNGS physics operation in August 2006. A second physics run was stopped after two days due to a leak on the reflector water cooling circuit. The origin for the leak has been identified and the repair is under way. During the physics run $\sim 8.5 \cdot 10^{17}$ protons on target (p.o.t.) were delivered. The average beam intensity was $\sim 1.4 \cdot 10^{13}$ p.o.t. per extraction, the maximum reached in 2006 was $1.75 \cdot 10^{13}$ p.o.t. per extraction.

The first muon detector station is very sensitive to misalignment between the target and the horn, the second station gives information on proton-target alignment [8]. Hence scanning the proton beam w.r.t. the target and the horn and comparing the muon monitor profiles yielded optimal secondary particle production efficiency and a precise alignment of the beam w.r.t the target and the horn, as described in [9].

The muon monitor reproducibility for all the detectors was measured to be better than $1 \%$ r.m.s. The response of the different muon monitors, as cross-checked with the motorised monitor, was found to be identical within $<1 \%$. Figure 5 shows the horizontal muon profiles in the two detector stations for two different target units. The target materials are similar: unit 1 is made of polycrystalline graphite; unit 3 is made of a carbon-carbon composite with a very similar density as the used graphite. Hence no 
difference in the particle production is expected. In addition we can conclude from the similar profiles of the two units, that the proton beam is well centred on the target, and that the mechanical positioning of the different target units is performed very accurately, as designed.

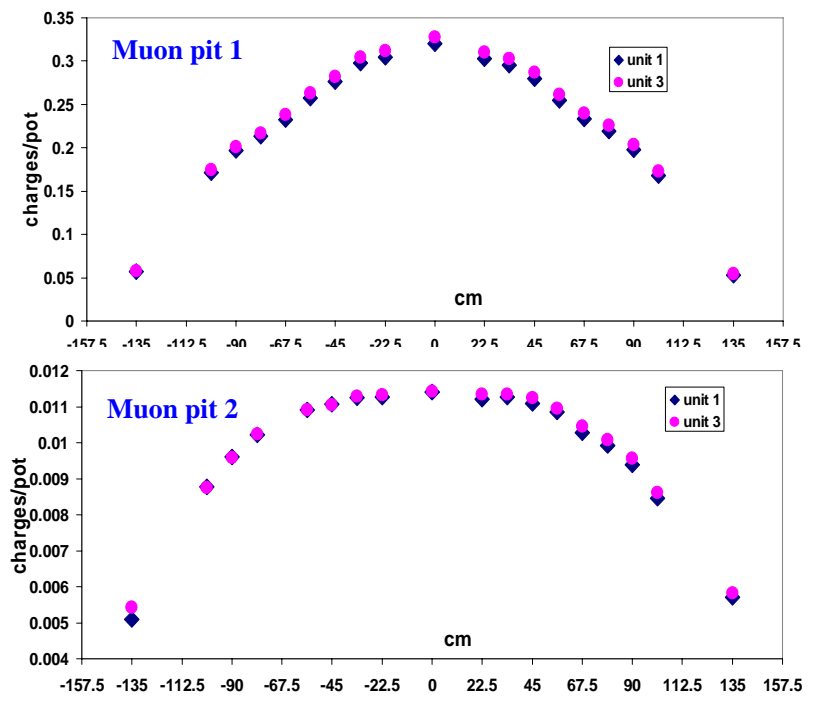

Figure 5: Horizontal muon monitor profiles for target unit 1 and unit 3. The results of the two measurements are almost identical. Each point corresponds to one detector and gives the charge per proton on target $(\sim 2700$ charges per muon).

The CNGS secondary beam contains only few elements which can be used to vary parameters and compare measurements with simulations. One possibility is to change the horn and reflector current. In Figure 6 the horizontal muon profile in the first detector station is shown for different horn and reflector current settings. The lowest set of data points stems from measurements with both horn and reflector OFF. The signal in the muon detectors is reduced by a factor $\sim 10$, as expected.

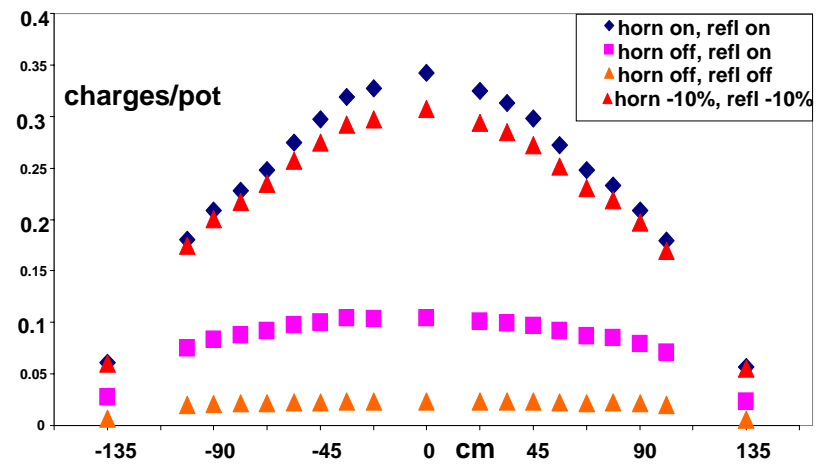

Figure 6: Horizontal muon profile in the first muon station for different horn and reflector field settings.

The CNGS muon profiles have been simulated with the FLUKA code $[10,11]$. The comparison of data with simulations is work in progress. A preliminary result is shown in Figure 7. The simulations agree very well with the measurements. However, further measurements (e.g. muon detector calibration in a dedicated muon beam experiment) and simulations, together with the operation of the CNGS facility at different settings, will be important to understand outstanding issues.

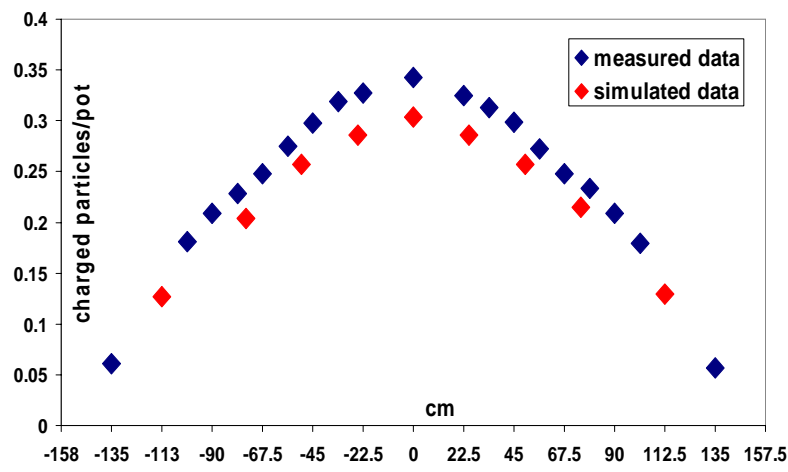

Figure 7: Measured horizontal muon monitor profile in the first muon station for optimised beam alignment, compared to simulations.

\section{SUMMARY}

The CNGS facility was successfully commissioned and operated for a first physics run in 2006. The performance in terms of neutrino production compares well with expectations from simulations. A further run is scheduled in autumn 2007.

The final benchmark for the design of the CNGS facility will be the continuous high intensity operation, scheduled to start in 2008. The resulting high radiation levels and fatigue effects from the pulsed operation and the beam impact will be a milestone to validate the design and reliability of the equipment, in particular of the target, horn and reflector.

\section{REFERENCES}

[1] 'General Description of the CNGS Project', 2000, http://proj-cngs.web.cern.ch/proj-cngs/

[2] M. Meddahi et al., PAC'07, Albuquerque, June 2007.

[3] L. Bruno et al., CERN-AB-2004-063 CNGS.

[4] L. Bruno, EDMS ${ }^{1}$ Id 772307.

[5] J.-M. Maugain et al., EDMS ${ }^{1}$ Id 383834, 383394, 485613, 481832, 481833, 607171, 787376, 772651.

[6] Ans Pardons, presentation given at NBI2006, http://indico.cern.ch/conferenceTimeTable.py?confId $=4371$.

[7] B. Dehning, EDMS1 Id 481835.

[8] A.E. Ball et al., CERN-SL-2001-016 EA

[9] E. Gschwendtner et al., NSS-IEEE06, San Diego, 2006, Report CERN-AB-2007-05 ATB.

[10] A. Fasso, A. Ferrari, P.R. Sala, , Proc. MonteCarlo 2000, Lisbon, 23-26 Oct. 2000, A. Kling et al. eds., Springer-Verlag Berlin, p.159-164 (2001).

[11] A. Fasso, A. Ferrari, J. Ranft, P.R. Sala, Proc. Monte Carlo 2000, Lisbon, 23-26 Oct. 2000, A. Kling, et al eds., Springer-Verlag Berlin, p.955-960 (2001).

1 access to EDMS: http://proj-cngs.web.cern.ch/proj-cngs/accessEDMS.htm 